\title{
Risk factors for breakthrough varicella in healthy children
}

\author{
Y J Lim, F T Chew, A Y S Tan, B W Lee
}

\begin{abstract}
Aim-To evaluate the risk factors for breakthrough varicella in a follow up study of a cohort of 181 healthy children immunised when aged 9-24 months with a reformulated Oka strain varicella vaccine (SmithKline Beecham Biologicals/Oka).

Design-The children were randomised in a double blind manner into one of four groups to receive one of two production lot vaccine batches, at two different titres (high titre, $10^{3.9}$ and $10^{4.0}$ plaque forming units (pfu); low titre (heat exposed), $10^{2.7}$ and $\left.10^{2.8} \mathrm{pfu}\right)$. The overall seroconversion rate after immunisation was $99 \%$.

Results-One hundred and sixty eight patients were available for review after a mean (SD) follow up of 35 (9) months after vaccination. Multivariate analysis indicated that risk factors for breakthrough varicella were household contact with varicella (adjusted odds ratio (OR), 19.89; $95 \%$ confidence interval (CI), 18.39 to 21.39 ), vaccination age of $\leqslant 14$ months (adjusted OR, 2.30; 95\% CI, 1.69 to 2.90 ), and receiving low titre $\left(10^{2.7} \mathrm{pfu}\right)$ vaccine (adjusted OR, 2.13; 95\% CI, 1.54 to 2.73 ). All children who developed breakthrough varicella, had a modified varicella illness, except for three, all of whom had received low titre vaccine.

Conclusion-The identification of young immunisation age ( $\leqslant 14$ months) and low titre vaccine as risk factors for breakthrough varicella have important implications for the implementation of varicella vaccination programmes in healthy children.

(Arch Dis Child 1998;79:478-480)
\end{abstract}

Keywords: varicella; vaccination

In recent years, varicella vaccination has become available for use in healthy children and adults in many communities. However, except for the USA, ${ }^{1}{ }^{2}$ Japan, and Korea, ${ }^{3}$ it has not been adopted as part of the routine childhood immunisation programme. The Oka strain varicella vaccine is highly immunogenic, ${ }^{4-8}$ and the current data indicate that immunity conferred by vaccination is long lasting, ${ }^{3910}$ especially with the presence of immunological boosting from circulating wild varicella virus in the community. However, there is still uncertainty about whether children immunised with varicella vaccine develop lifelong immunity. ${ }^{11}$ Furthermore, the widespread use of this vaccine and its introduction into childhood immunisation programmes is likely to reduce the incidence of varicella and, therefore, also reduce boosting of immunity in vaccinees.

Reports on the short term protective efficacy of the varicella vaccine varies from $<70 \%$ to $>96 \%{ }^{4}$ Fortunately, varicella in immunised individuals (breakthrough varicella) is modified and much less severe than the natural disease..$^{6-812} 13$ In our study, a follow up evaluation of a cohort of young healthy children who participated in a clinical trial involving the use of a reformulated Oka strain varicella vaccine (SmithKline Beecham Biologicals/Oka) was carried out to assess the possible factors associated with breakthrough varicella.

\section{Materials and methods}

VACCINES

The varicella vaccine used was a reformulated Oka strain vaccine (Oka/SmithKline Beecham Biologicals, Rixensart, Belgium) with a stabiliser to provide a shelf life of up to two years when stored at $2-8^{\circ} \mathrm{C} .{ }^{5}$ Table 1 shows the vaccine lots and corresponding titres of the vaccines used. The heat exposed vaccine lots VA104B42/7 and VA107A42/10 were obtained by exposure of each lot, VA104B42 and VA107A42, to a temperature of $37^{\circ} \mathrm{C}$ for seven and 10 days, respectively. ${ }^{14}$

\section{CLINICAL TRIAL}

The vaccine was administered in a double blind randomised manner. Healthy children aged 9 to 24 months were recruited between September 1992 and March 1994. The reactogenicity and immunogenicity (seroconversion of $98.9 \%$ ) of the vaccines used in this trial has been reported. ${ }^{14}$ Assessment of seroconversion at six weeks after vaccination was carried out by measurement of varicella specific antibodies using the indirect immunofluorescence method. ${ }^{15}$

On two separate occasions after completion of the trial, between August and September 1994, and April and May 1996, a follow up evaluation of the vaccinees for a history of breakthrough varicella was made by a telephone interview with the parents to inquire about a history of contact with wild varicella and the possibility of breakthrough varicella in the vaccinee. A contact history of varicella was

Table 1 Vaccine lots and titres used

\begin{tabular}{lll}
\hline Group & Vaccine lots & pfu/dose \\
\hline 1 & VA104B42 & $10^{3.9}$ \\
2 & VA104B42/7 & $10^{2.7}$ \\
3 & VA107A42 & $10^{4.0}$ \\
4 & VA107A42/10 & $10^{2.8}$ \\
\hline
\end{tabular}


Table 2 Type of contact history and incidence of breakthrough varicella

\begin{tabular}{llll}
\hline Type of contact & Patients (n) & Breakthrough varicella & Adjusted OR (95\% CI) \\
\hline Household & 47 & 17 & 19.89 (18.39 to 21.39) \\
Creche & 32 & 1 & NS \\
Visiting & 18 & 0 & NS \\
\hline
\end{tabular}

Fifteen patients had more than one contact. Reference category of each case in the multiple logistic regression was the non-exposed (or no contact) group.

defined as exposure to a person with varicella for at least four hours. ${ }^{14}$ Contact was classified as household, creche, or visiting relatives/ friends. For those with a history of breakthrough varicella, the diagnosis was made by examination by the investigators or by a family physician. Mild or modified varicella illness was described as an illness with an estimate of fewer than 50 vesicular lesions. ${ }^{16}{ }^{17}$ None of the breakthrough varicella illnesses was confirmed by serology.

\section{STATISTICAL ANALYSIS}

The data were analysed initially by cross tabulation using the $\chi^{2}$ test or Fisher's exact test (where appropriate). Multiple logistic regression analysis was then used to control potential confounding factors and to select the significant independent risk factors associated with breakthrough varicella. This was done by computing the adjusted odds ratios (OR) and 95\% confidence intervals (95\% CI). Statistical analysis was carried out using the PROC FREQ and PROC LOGISTIC procedures of the statistical package SAS (version 6.08) for personal computers.

\section{Results}

Of the 181 patients who completed the initial 42 day follow up of the clinical trial, 168 patients ( 80 girls, 88 boys) could be contacted after a mean (SD) follow up period of 35 (9) months. Two children who did not seroconvert and were subsequently given booster immunisations were excluded from the analysis. Eleven children were lost to follow up. Eighty two $(49 \%)$ had a history of exposure (as defined by at least four hours' exposure) to varicella. Only children with a known contact with persons with natural varicella were reported to have had breakthrough varicella. These illnesses oc-

Table 3 Factors associated with breakthrough varicella

\begin{tabular}{|c|c|c|c|c|c|}
\hline Factor & $n$ & Contact history & Breakthrough varicella & Adjusted $O R$ & $95 \% C I$ \\
\hline \multicolumn{6}{|l|}{ Vaccination age ${ }^{\star}$} \\
\hline$\leqslant 14$ months & 60 & 26 & 10 & 2.30 & \multirow[t]{2}{*}{1.69 to 2.90} \\
\hline$>14$ months & 108 & 56 & 8 & 1 & \\
\hline \multicolumn{6}{|l|}{ Vaccine titre $†$} \\
\hline Group 1 & 43 & 18 & 3 & NS & \multirow{4}{*}{1.54 to 2.73} \\
\hline Group 2 & 42 & 18 & 8 & 2.13 & \\
\hline Group 3 & 40 & 22 & 2 & NS & \\
\hline Group 4 & 43 & 24 & 5 & NS & \\
\hline \multicolumn{6}{|l|}{$V_{z A b^{\star} \ddagger}$} \\
\hline$<64^{+}$ & 67 & 25 & 5 & 1 & \\
\hline$\geqslant 64$ & 101 & 57 & 13 & NS & \\
\hline \multicolumn{6}{|l|}{ Sex } \\
\hline Female & 80 & 36 & 8 & NS & \\
\hline Male & 88 & 46 & 10 & 1 & \\
\hline \multicolumn{6}{|c|}{ Follow up period (months) } \\
\hline$\leqslant 37.175$ & 84 & 39 & 9 & 1 & \\
\hline$>37.175$ & 84 & 43 & 9 & NS & \\
\hline
\end{tabular}

${ }^{\star}$ A cut off age of 14 months and a titre of $1 / 64$ were used as these were the medians for the cohort. tReference category for vaccine titre in each case in the multiple logistic regression was the other titres (for example, reference category for group 1 in the comparison was groups $2-4$ ).

$\ddagger$ VzAb denotes the varicella specific antibody titre six weeks after immunisation. ${ }^{14}$ curred two to three weeks after exposure and were verified by a doctor. There were 18 children with breakthrough varicella illness. Table 2 summarises the type of contact history and incidence of breakthrough varicella.

To evaluate the factors associated with breakthrough varicella, multiple logistic regression analysis was carried out. The factors included in this analysis included the age of vaccination, sex of subject, type of varicella contact, vaccine received (groups 1-4, table 1), postvaccination varicella antibody titres, and length of follow up. Tables 2 and 3 summarise the results of the analysis. The significant factors associated with breakthrough varicella were household contact, vaccination age of $\leqslant 14$ months, and receiving the lowest titre heat exposed vaccine (group 2).

When the cut off age for this analysis was set at 12 months, the youngest age recommended by the American Academy of Paediatrics for varicella immunisation in healthy children, ${ }^{1}$ the age of vaccination remained a significant factor (adjusted OR, 2.21;95\% CI, 1.65 to 2.77 ). When the cut off age was set above 14 months age was not a significant factor (data not shown).

In addition, when the data were analysed by high and low titre vaccine lots (high titre, groups 1 and 3; low titre, groups 2 and 4) (table 1 ), the low titre vaccine was associated significantly with breakthrough disease (adjusted OR, $1.60 ; 95 \%$ CI, 1.03 to 2.17 ). The varicella antibody titre remained not significant even when analysis was carried out with various antibody titre cut off points $(1 / 16,1 / 32,1 / 64)$.

Of the 18 children with breakthrough varicella, 15 had mild illness; the three children who developed significant varicella illness (generalised vesicular rash) were given the low titre heat exposed vaccine. These three children were aged 10,12 , and 17 months at the time of vaccination.

\section{Discussion}

The Oka strain varicella vaccine is a well tolerated and highly immunogenic vaccine that has recently become more widely available for the immunisation of healthy children. ${ }^{13910}$ Our study involved a cohort of 181 children aged between 9 and 24 months, who participated in a clinical trial and were randomly selected to receive immunisation with one of two production lots of vaccine at two different vaccine titres (high release and lower heat exposed titres) (table 1). Our results indicate that the vaccine was highly immunogenic $(98.9 \%$ seroconversion) and well tolerated. ${ }^{14}$ Although the geometric mean titres of varicella specific antibodies were lower for those who received the heat exposed low titre vaccine, comparison with the groups receiving high titre vaccine showed no significant differences. ${ }^{14}$ This clinical trial also provided us with the opportunity to undertake a follow up study of this cohort to evaluate breakthrough varicella and identify the factors associated with it.

After a mean follow up of 35 months, 18 of 82 children who had a history of varicella contact were reported to have had breakthrough disease. Krause reported a similar breakthrough rate 
$(20 \%)$ for vaccinated children after household contact. ${ }^{4}$ This contrasts with the high rate of $87 \%$ in unvaccinated children contracting the disease after household contact, ${ }^{18}$ and indicates protection against the disease. Household varicella contact is an important factor in the spread of this contagious infection, and all but one of the children in this study developed breakthrough disease through this means of contact with the wild virus.

In this study, the diagnosis of breakthrough illness was made only on clinical grounds, and was not confirmed serologically. In addition, only two telephone contacts with parents/ guardians were made throughout the entire period of follow up. However, an accurate clinical diagnosis was made by medical personnel and the history of varicella contact history in all the cases supported this diagnosis. Nevertheless, it is possible that very mild breakthrough illnesses might have been missed, although these would probably not have been clinically relevant.

Of interest, multiple logistic regression analysis indicated that breakthrough varicella illness was significantly associated with a younger age of administration ( $\leqslant 14$ months) and with those receiving the heat exposed low titre vaccine lots. Similar results with regard to the association between low titre vaccine and breakthrough varicella were reported by Varis and Vesikari. ${ }^{19}$ In their study, two production lots of SmithKline Beecham Biologicals/Oka reformulated vaccine, similar to those used in our study, were administered to a cohort of Finnish children (10-30 months old). The vaccine titres of the heat exposed vaccine lots were only marginally higher than those in our study (Finnish study, $10^{3.1}$ plaque forming units (pfu)/dose and $10^{2.8} \mathrm{pfu} /$ dose; our study, $10^{2.8}$ $\mathrm{pfu} /$ dose and $10^{2.7} \mathrm{pfu} /$ dose). Higher rates of breakthrough varicella disease have also been associated with lower titre vaccines in studies carried out in the USA with varicella vaccines that have not been formulated to confer better heat stability. ${ }^{416} 20$

Varis and Vesikari also reported that the vaccine efficacy was lowest in the younger age group (10-18 months), although statistical analysis was not carried out in their study. ${ }^{19}$ This observation is likely to be related to interference by maternal antibodies in the younger vaccinees, or lower cell mediated immune responses to varicella vaccine in very young children. ${ }^{21}$ Our data suggest that vaccine efficacy is significantly better in those vaccinated $\geqslant 14$ months of age. It would be important to verify this observation in a larger cohort, because the current recommended age for varicella vaccination is $\geqslant 12$ months, ${ }^{1}$ and this may, therefore, have to be reviewed.

As seen in our study, breakthrough varicella is generally milder than the natural disease in children. ${ }^{3781213}$ However, it was noted that three of the 18 children in our cohort with breakthrough disease had generalised vesicles, indistinguishable from those seen in unvaccinated children. All three of these children had received the heat exposed low titre vaccine.
Although the breakthrough varicella rate in our cohort of children was 18 of 82 , only one case of breakthrough varicella occurred in the children who had been vaccinated with the vaccine that complied with current marketed vaccine titres for the reformulated vaccine (expiry titre not $<10^{3.3} \mathrm{pfu} /$ dose) and who were the age currently recommended for vaccination (> 12 months). Thus, in conclusion, age of vaccination and vaccine titre appear to be important factors in the occurrence of breakthrough varicella after immunisation. In addition, although breakthrough cases of varicella in vaccinees are generally mild, these factors should be taken into consideration when implementing a vaccination programme for healthy children. Because latent infection is likely to be related to the skin lesions seen in varicella, reducing breakthrough varicella in those vaccinated might also play a role in decreasing the incidence of herpes zoster after vaccination. $^{22} 23$

1 Committee on infectious diseases. American Academy of Pediatrics. Recommendations for the use of live attenuated varicella vaccine. Pediatrics 1995;95:791-6.

2 Gershon AA. Varicella vaccine: its past, present and future. Pediatr Infect Dis $\mathcal{F}$ 1995;14:742-4.

3 Asano Y. Varicella vaccine: the Japanese experience. $\mathcal{F}$ Infect Dis 1996;174(suppl 3):310-13.

4 Krause PR, Klinman DM. Efficacy, immunogenicity, safety, and use of live attenuated chickenpox vaccine. $\mathcal{F}$ Pediatr 1995; 127:518-25.

5 Meurice F, DeBouver JL, Vandevoorde D, Woods S, Bogaerts H. Immunogenicity and safety of a live attenuated varicella vaccine (Oka/SB Bio) in healthy children. F Infect Dis 1996;174(suppl 3):324-9.

6 Weibel R, Neff BJ, Kuter BJ, et al. Live attenuated varicella virus vaccine: efficacy trial in healthy children. $N$ Eng $\mathcal{F} M e d$ 1984;310:1409-15.

7 White CJ, Kuter BJ, Hildebrand CS, et al. Varicella vaccine (VARIVAX) in healthy children and adolescents: results from clinical trials, 1987 to 1989 . Pediatrics $1991 ; 87: 604-$ 10.

8 White CJ, Kuter BJ, Ngai A, et al. Modified cases of chickenpox after varicella vaccination: correlation of protection

with antibody response. Pediatr Infect Dis f 1992;11:19-23. immunity of recipients of the Oka strain of live varicella immunity of recipients of the Oka

10 Asano Y, Suga S, Yoshikawa T, et al. Twenty-year follow-up of protective immunity of the Oka strain live varicella vacof protective immunity of the Oka
cine. Pediatrics 1995;94:524-6.

11 Ross LF, Lantos JD. Immunisation against chickenpox: better to confine immunisation to those at high risk. BMF 1995;310:2-3

12 Bernstein $\mathrm{HH}$, Rothstein EP, Watson BM, et al. Clinical survey of natural varicella compared with breakthrough varicella after immunization with live attenuated Oka/ Merck varicella vaccine. Pediatrics 1993;92:833-7.

13 Watson BM, Piercy SA, Plotkin SA, Starr SE. Modified chickenpox in children immunized with the Oka/Merck varicella vaccine. Pediatrics 1993;91:17-22.

14 Tan AYS, Connett CJ, Connett GJ, et al. Use of a reformulated Oka strain varicella vaccine (SmithKline Beecham Biologicals/Oka) in healthy children. Eur 7 Pediatr 1996; 155:706-11.

15 Leventon-Kriss S, Yoffe R, Rannos L, Modan M. Seroepidemiologic aspects of varicella zoster virus infections in an Israeli Jewish population. Isr f Med Sci 1978;14:766-70.

Israeli Jewish population. Isr F Med Sci 1978;14:766-70.
16 White CJ. Clinical trials of varicella vaccine in healthy chilWhite CJ. Clinical trials of varicella vaccine in he
dren. Infect Dis Clin North Am 1996;10:595-608.

dren. Infect Dis Clin North Am 1996;10:595-608.
17 White CJ. Varicella-zoster virus vaccine. Clin Infect Dis 1997;24:753-63.

18 Ross AH, Lenchner E, Reitman G. Modification of chickenpox in family contacts by administration of gamma globulin. N Engl fै Med 1962;267:369-76.

19 Varis T, Vesikari T. Efficacy of high-titer live attenuated varicella vaccine in healthy young children. F Infect Dis 1996;174(suppl 3):S330-4.

20 Ellis RW. Varicella vaccine. Vaccine 1995;13:1387-8.

21 Terada K, Kawano S, Yoshihiro K, Morita T. Varicellazoster virus (VZV) reactivation is related to the low response of VZV-specific immunity after chickenpox in infancy. F Infect Dis 1994;169:650-2

22 Hardy IB, Gershon A, Steinberg S, LaRussa P. NIAID collaborative varicella vaccine study group. The incidence of
zoster after immunization with live attenuated varicella vaccine. A study in children with leukemia. $N$ Engl $\mathcal{F}$ Med 1991;325:1545-50.

23 Levin MJ, Hayward AR. The varicella vaccine. Prevention of herpes zoster. Infect Dis Clin North Am 1996;10:657-75. 